\title{
Clinical Value of the First Dedicated, Commercially Available Automatic Injector for Ictal Brain SPECT in Presurgical Evaluation of Pediatric Epilepsy: Comparison with Manual Injection
}

\author{
Sunhee Kim ${ }^{1}$, Deborah L. Holder ${ }^{2}$, Charles M. Laymon ${ }^{3}$, Dana L. Tudorascu ${ }^{4}$, Erin L. Deeb ${ }^{3}$, Ashok Panigrahy ${ }^{1}$, \\ and James M. Mountz ${ }^{3}$ \\ ${ }^{1}$ Department of Radiology, Children's Hospital of Pittsburgh of UPMC, Pittsburgh, Pennsylvania; ${ }^{2}$ Department of Pediatric \\ Neurology, Children's Hospital of Pittsburgh of UPMC, Pittsburgh, Pennsylvania; ${ }^{3}$ Department of Radiology, University of Pittsburgh, \\ Pittsburgh, Pennsylvania; and ${ }^{4}$ Department of Medicine and Biostatistics, University of Pittsburgh, Pennsylvania
}

\begin{abstract}
The most challenging technical problem in ictal brain SPECT for localization of an epileptogenic focus is obtaining a timely injection of a radiopharmaceutical. In our institution, the first dedicated commercially available, remotely controlled automatic injector has been used in the pediatric epilepsy unit in conjunction with 24-h video and electroencephalogram monitoring. The goal of this study was to demonstrate the improved success rate of ictal injection by use of the automatic injector in the pediatric population. Methods: Eighty-four pediatric patients and eighty-four $99 \mathrm{mTc}$-ethylcysteinate dimer (99mTcECD) ictal brain SPECT studies were retrospectively analyzed in a masked manner. The group with manual injection consisted of 45 studies performed from 2004 to 2010 before the introduction of the automatic injector. The group with automatic injection consisted of 39 studies performed from 2010 to 2011 after the introduction of the automatic injector. The 2 groups were comparable in the total duration of seizure, injected dose, and time from the injection to the image acquisition. The latency time from the seizure onset to the initiation time of injection, the ratio of latency time to total duration of seizure $(\mathrm{L} / \mathrm{T})$, the number of patients with repeated studies, the number of days of additional hospitalization for each study, and the localization rate for identifying a single focus in each study were compared between the groups. Results: The median latency time in the group with automatic injection (8 s) was significantly lower than that of the group with manual injection (18 s) $(P<0.05)$. Also there was a statistically significant decrease in the number of patients with repeated studies in the group with automatic injection $(2 / 39$ [5\%]), compared with the group with manual injection (14/45 [31\%]) $(P<0.05)$. The median number of days of additional hospitalization in the group with manual injection (range, 0-7) was statistically significantly different, compared with the group with automatic injection (range, $0-1)(P<$ 0.05). In the group with automatic injection, 31 of 39 scans
\end{abstract}

Received Jul. 30, 2012; revision accepted Nov. 26, 2012.

For correspondence or reprints contact: James M. Mountz, Division of Nuclear Medicine, Department of Radiology, University of Pittsburgh Medical Center, PET

Facility, B-932, 200 Lothrop St., Pittsburgh, PA 15213.

E-mail: mountzjm@upmc.edu

Published online Mar. 14, 2013.

COPYRIGHT @ 2013 by the Society of Nuclear Medicine and Molecular Imaging, Inc. demonstrated a single localizing focus, compared to 22 of 45 scans from the manual-injection group, a significant difference $(P<0.05)$. The radiation exposure rate to nursing staff during the periods with automatic injection was lower than during the periods with manual injection. Conclusion: The automatic injector combined with 24-h video and electroencephalogram monitoring demonstrated significant clinical value by decreasing latency time, the number of patients with repeated studies, and the number of days of additional hospitalization while increasing the number of studies with a single localizing focus.

Key Words: automatic injector; pediatric; epilepsy; ictal SPECT

J Nucl Med 2013; 54:732-738

DOI: 10.2967/jnumed.112.105189

O the quality of life of epilepsy patients. Systematic reviews reported that $66 \%-70 \%$ of patients were seizure-free at short-term ( $<5$ y) follow-up $(1-4)$, and $41 \%-79 \%$ were seizurefree at long-term $(>5$ y) follow-up after temporal lobe resection (5-8). Successful surgery depends on the accurate localization of epileptogenic focus.

The utility of scalp electroencephalography for accurate localization is constrained by the following factors: the test records only surface cortical activity, it provides limited sampling of the brain, and it has low spatial resolution. An intracranial electroencephalogram can detect signals from deeper brain structures but is useful only if electrode coverage includes the epileptogenic zone. Electrode coverage is limited by invasiveness and risk of surgical complications (9).

Imaging modalities such as SPECT, PET, and MR imaging allow for noninvasive whole-brain, epilepsy evaluation presurgically. Among those modalities, ictal SPECT is the only imaging modality that allows identification of the ictal onset zone by measuring regional cerebral blood 
flow at the time of injection (10-13). Increased perfusion is expected within that ictal onset zone if the injection occurs before seizure propagation. Previous studies including metaanalysis demonstrated that ictal SPECT had the highest sensitivity among imaging modalities in identifying epileptogenic focus in both temporal lobe (90\%-100\%) and extratemporal lobe epilepsy (81\%) (14-16).

More recently, subtraction ictal SPECT techniques and statistical comparison with normal control SPECT databases have been validated by several studies, showing a rate of epileptogenic focus localization that was equivalent or superior to that seen in interictal-ictal side-by-side visual analysis $(17-21)$.

The diagnostic quality and resultant accuracy of ictal SPECT and ictal-interictal SPECT image subtraction are significantly increased by rapid ictal injection after seizure onset. The capability for reliable prompt injection requires meticulous coordination between the neurologic epilepsy team and nuclear medicine facility. The initiation of ictal SPECT is based on continuous video electroencephalogram monitoring. The most common practice today is to have trained epilepsy staff physicians or electroencephalogram technologists continuously monitor the patient's video electroencephalogram, often near the patient's bedside, and perform an immediate manual injection of radiotracer on seizure onset. The limitation of this type of manual injection is the delay time (latency) between seizure onset and actual injection time. Time delays can occur during the notification of seizure onset, opening of the lead-shielded radiotracer container, removal of the syringe, and insertion of the syringe into the intravenous line port, and between tracer injection and flush of the intravenous line. Although these delays may take only a relatively short time with experienced personnel, they can make a significant difference in seizures of relatively short duration. Thus, the difficulties in obtaining successful and early infusion of the tracer have limited the quality of ictal SPECT (22).

To solve this technical challenge, a custom-built automated injection system was introduced in the previous study by Sepkuty et al (23). The automatic injector was designed to reduce the latency time of injection from the seizure onset to injection by allowing personnel who first recognize the seizure activity to inject the radiopharmaceutical remotely (23). On the basis of their in vitro experiments, Sepkuty et al showed that their automatic injection system, compared with manual injection, shortened latency time; however, this system was custom-made and not commercially available.

Later, Feichtinger et al. published on a modified, remotely controlled automatic CT contrast agent injection system designed for ictal SPECT tracer application (Medrad Vistron CT injection system; Medrad Inc.) and reported its efficacy and safety based on an evaluation in 26 patients but without comparison to manual injection (24).

Recently, Setoain et al. demonstrated their custom-built automatic injector and compared its performance with manual injection (25). Although they found significant reduction in time from the seizure onset to the end of radiopharmaceutical injection, they did not find statistically significant improvement in the detection rate of seizure foci.

The first commercially available, dedicated remotely controlled automatic injector (Spectris Solaris EP; Medrad Inc.) has been used in our pediatric epilepsy unit in conjunction with 24-h video and electroencephalogram monitoring. In this paper, we report the first, to our knowledge, results on the clinical value of the dedicated automatic injector with a retrospective comparison with manual injections for the presurgical evaluation of pediatric epilepsy.

\section{MATERIALS AND METHODS}

\section{Patients}

Eighty-four ${ }^{99 \mathrm{~m}}$ Tc-ethylcysteinate dimer (99m Tc-ECD) ictal brain SPECT studies in 84 pediatric patients were retrospectively reviewed after approval from the institutional review board to review patient data through an honest-broker system was obtained. The manual-injection group consisted of 45 pediatric patients (median age, 12 y [age range, 3-18 y]; 24 males and 21 females) who underwent 61 ictal SPECT studies performed with the manual injector from 2004 to 2010 . After repeated studies in a subject were excluded, 45 studies were collected. The automatic-injection group consisted of 39 patients (median age, $10 \mathrm{y}$ [age range, 2-19 y]; 20 male and 19 female) who underwent 41 ictal SPECT studies performed with the automatic injector from 2010 to 2011. After repeated studies in a subject were excluded, 39 studies were collected. Patient demographics are shown in Table 1.

\section{Ictal SPECT Injection Procedure}

Manual Injection. All SPECT studies performed before March 2010 had manual intravenous injections of ${ }^{99 m}$ Tc-ECD. As soon as a nurse monitoring a patient was notified by electroencephalogram technologists, the radiotracer, stored in a shielded box, was injected directly into the saline lock of the intravenous access to the patient. The total volume of radiopharmaceuticals was $1 \mathrm{~mL}$ and was injected within a period of $1 \mathrm{~s}$ manually, followed by 10 $\mathrm{mL}$ of saline flush.

Automatic Injection. The automatic injector was introduced after March 2010. The radiopharmaceutical, ${ }^{99 m} \mathrm{Tc}-\mathrm{ECD}$, was prepared in a volume of $7 \mathrm{~mL}$ and loaded twice a day in a shielded syringe within the injector pump by a nuclear medicine technologist (Fig. 1). The radiotracer vial was placed in the automatic injector at $7 \mathrm{AM}$ and calibrated for 1,665 MBq (45 mCi) at 11:30 AM. At 11:30 AM, a second radiotracer vial replaced the first and was calibrated for $1,665 \mathrm{MBq}(45 \mathrm{mCi})$ at 4:30 PM. The radiotracer loaded in the injection pump was then transported to the patient's room and connected with the patient's intravenous access using a thin tube system (length $243 \mathrm{~cm}$; total volume, $7 \mathrm{~mL}$ ). This tube system allowed patients free mobility, especially during the seizure. When the electroencephalogram technologists recognized seizure activity either on the electroencephalogram strip or in the video, they pushed the start button of the remote control panel, initiating automatic injection of the $7 \mathrm{~mL}$ of radiopharmaceutical, followed by an automatic $20-\mathrm{mL}$ saline flush. The radiotracer and flush were delivered at a rate of $2 \mathrm{~mL} / \mathrm{s}$. 
TABLE 1

Patient Demographics

\begin{tabular}{lcc}
\hline \multicolumn{1}{c}{ Demographic } & Group with manual injection & Group with automatic injection \\
\hline Period of data collection & June 2004 to February 2010 & March 2010 to September 2011 \\
Age (y) & 12 \\
Median & $3-18$ \\
Range & 24 \\
Sex & 21 \\
Male & 20 \\
Female & 65 \\
Duration of seizure (s) & 19 \\
Median & $2-1,005$ \\
Range & $2-753$ \\
\hline
\end{tabular}

\section{Seizure Parameters}

All 84 patients underwent continuous video electroencephalogram monitoring. The onset and termination time of each seizure was determined using the video electroencephalogram recording. The onset of seizures, time of injection of radiopharmaceuticals, and termination time of seizures were recorded by electroencephalogram technologists and confirmed by epileptologists.

The total duration of seizure was defined as the time from the onset of seizure to the time of termination of seizure. The latency time was defined as the time delay from the onset of seizure to the initiation time of the radiopharmaceutical injection.

\section{SPECT Methods}

Within an hour of the radiopharmaceutical injection, the patient was stabilized and transported to the nuclear medicine division. The patient was placed supine, with a headholder to restrict head motion and a pillow beneath the knees for comfort. Under certain circumstances, sedation was required to control patient movement; sedation use was arranged in advance with the epilepsy monitoring unit and coordinated with the radiology anesthesiologist and radiology nursing staff. If sedation was performed, it was administered at least $30 \mathrm{~min}$ after tracer injection.

Ictal SPECT scans were obtained using a large-field-of-view dual-head $\gamma$-camera (Infinia Hawkeye; GE Healthcare) equipped with low-energy all-purpose collimators and acquired into a $128 \times$ 128 matrix using 128 total projections at $40 \mathrm{~s} /$ projection. Image thickness was $3 \mathrm{~mm}$ per slice. Images were reconstructed using ordered-subset expectation maximization and then sectioned in the transverse, sagittal, and coronal planes for visual assessment.

\section{Scan Assessment}

Each ictal SPECT scan was assessed for the presence of a single localizing focus (a focus of tracer uptake with more intensity than adjacent gray matter activity and more intensity relative to baseline). No localizing focus was defined when there was no single identifiable focus or multiple areas of foci of tracer uptake with more intensity than adjacent gray matter activity and more intensity relative to baseline. Two reviewers assessed all images in an independent and masked manner. There were discrepant reads in 2 cases, which were subsequently evaluated by the 2 reviewers; consensus was reached in both cases.

\section{Repeated Studies}

Video electroencephalogram, seizure semiology, ictal and interictal SPECT, MR imaging, and other relevant clinical information were reviewed by radiologists, nuclear medicine physicians, and epileptologists shortly after the completion of ictal SPECT. On the basis of this review, a consensus was reached as to whether a repeated ictal SPECT scan was necessary.

FIGURE 1. Automatic injector. (A) Injector with loading syringes. Syringe on right side is loaded with radiopharmaceutical and syringe on left is loaded with intravenous fluid for KVO (keep vein open) and flush. (B) Remote monitor, which will program and store protocols.

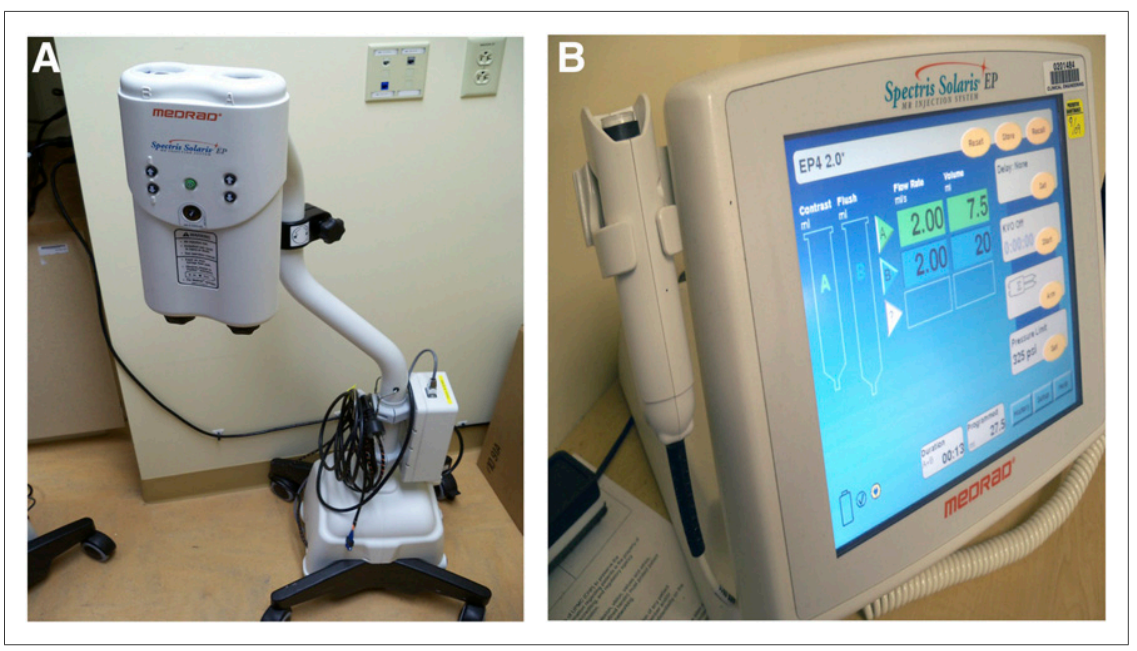


TABLE 2

Comparison of Parameters Between Groups with Manual Injection and Automatic Injection

\begin{tabular}{|c|c|c|c|}
\hline Parameter & $\begin{array}{l}\text { Group with manual injection } \\
\qquad(n=45 \text { studies })\end{array}$ & $\begin{array}{l}\text { Group with automatic injection } \\
\text { ( } n=39 \text { studies) }\end{array}$ & $P$ \\
\hline Latency time (s) & & & 0.00004 \\
\hline Median & 18 & 8 & \\
\hline Minimum & 2 & 2 & \\
\hline Maximum & 85 & 22 & \\
\hline $\mathrm{L} / \mathrm{T}$ ratio & & & 0.042 \\
\hline Median & 0.29 & 0.24 & \\
\hline Minimum & 0.02 & 0.01 & \\
\hline Maximum & 1.85 & 1 & \\
\hline No. of patients with repeated study & $14(31 \%)$ & $2(5 \%)$ & 0.003 \\
\hline Range of days of additional hospitalization & $0-7$ & $0-1$ & 0.002 \\
\hline
\end{tabular}

The same manner of decision-making was performed for the automatic- and manual-injection groups. The number of patients with repeated studies and number of days of additional hospitalization for each study were recorded.

\section{Data Analysis}

A Wilcoxon rank sum test was used to examine the significance of differences between the manual- and automatic-injection groups of total duration of seizure, latency time of injection, L/T ratio, and number of days of additional hospitalization for each study. $\chi^{2}$ tests were used for comparison of parameters such as the number of studies with and without a single localizing focus and the number of patients who had repeated studies between the 2 groups.

Radiation exposure to the unit nursing staff was investigated using the radiation dosimetry reports generated from analysis of thermoluminescent dosimeters over a 2-y period (March 2009 to February 2011 [March 2009 to February 2010 for manual injection and March 2010 to February 2011 for automatic injection]). During this time, 8 subjects and 11 studies were evaluated using the manual-injection procedure and 22 subjects and 23 studies using automatic-injection procedure. Staff were requested to wear their thermoluminescent dosimeters at chest level. Dosimeters were exchanged monthly, and doses below $1 \mathrm{mrem}$ (minimal dose) were not reported. The total reported dose to the nursing staff during the manual- and automatic-injection periods was calculated. The staff dose per patient treated and per study was calculated by dividing the total reported dose during each period by the number of patients monitored and by the number of studies, respectively.

\section{RESULTS}

Group differences in ictal SPECT timing parameters, repeated scans, and hospital-stay lengths are summarized in Table 2.

\section{Ictal SPECT Timing Parameters}

Total Duration of Seizure. Both the manual-injection and the automatic-injection groups had similar duration of seizure, with a median of 65 and $67 \mathrm{~s}$, respectively. Using a Wilcoxon rank sum test, we detected no statistically significant difference in seizure duration between the 2 groups $(P=0.59)$.

Latency Time from Seizure Onset to Initiation Time of Injection. The manual-injection group had significantly longer median latency time (18 s) than the automatic-injection group $(8 \mathrm{~s})$, with a $P$ value of 0.00004 based on Wilcoxon rank sum test.

L/T Ratio. The manual-injection group had a median L/T ratio of 0.29 , and the automatic-injection group had a median $\mathrm{L} / \mathrm{T}$ ratio of 0.19 . On the basis of the Wilcoxon rank sum test, the L/T ratio was found to be significantly different between the 2 groups $(P=0.042)$.

\section{Ictal SPECT Image Assessment}

The ictal SPECT image assessment intergroup comparison is summarized in Table 3.

The manual-injection group exhibited a significantly lower fraction of studies with a single localizing focus

TABLE 3

Comparison of Localization Rate by Identification of Single Localizing Focus

\begin{tabular}{lcc}
\hline No. of studies with... & $\begin{array}{c}\text { Group with manual injection } \\
(n=45 \text { studies })\end{array}$ & $\begin{array}{c}\text { Group with automatic injection } \\
(n=39 \text { studies })\end{array}$ \\
\hline No localizing focus ${ }^{*}$ & 23 & 8 \\
A single localizing focus & 22 & 31 \\
& & \\
\hline${ }^{*}$ No localizing focus includes scan with no single localizing focus or scan with multiple foci of increased tracer uptake.
\end{tabular}


FIGURE 2. Ictal and interictal SPECT scans with manual injection in 3-y-old male with intractable epilepsy. (A) Interictal SPECT images demonstrate diffuse prominent hypoperfusion involving left frontotemporal region. (B) First ictal SPECT attempt. Injection was performed $40 \mathrm{~s}$ after onset of seizure. Seizure duration was $46 \mathrm{~s}$, and therefore this late ictal SPECT scan demonstrates hypoperfusion in left frontotemporal region, with similar perfusion pattern seen in interictal SPECT. (C) Repeated ictal SPECT attempt. Injection was performed $2 \mathrm{~s}$ after onset of seizure. Seizure duration was $9 \mathrm{~s}$, and therefore this early ictal SPECT scan demonstrates moderate, relative increase in perfusion in left frontotemporal region, compared with right. Findings show significant change in perfusion pattern, compared with postictal and interictal SPECT, demonstrating the value of an early injection.

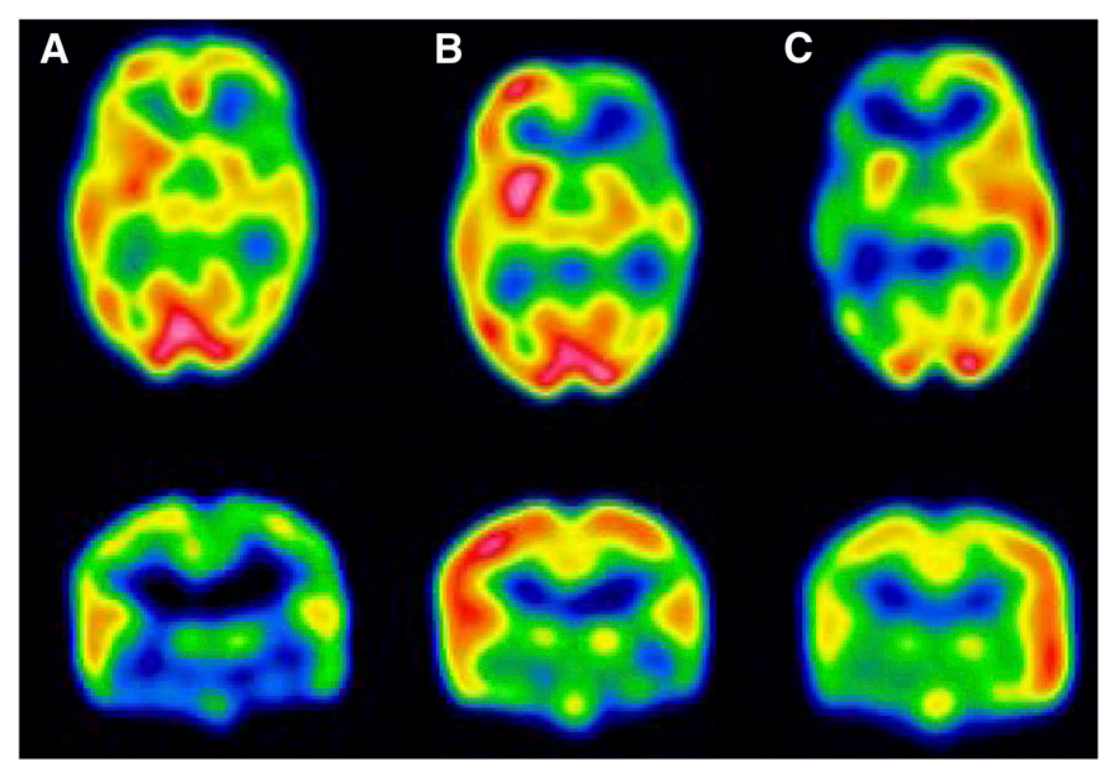

(22/45 studies) than the automatic-injection group (31/39 studies). Similarly, the manual-injection group had a significantly greater fraction of studies with no localizing focus (23/45 studies) than the automatic-injection group (8/39 studies).

Using the $\chi^{2}$ test, we found the difference in the fraction of studies with a localizing focus between the groups to be statistically significant $(P=0.0075$, Table 3$)$. Figures 2 and 3 are representative ictal and interictal SPECT images obtained by manual and automatic injection, respectively.

\section{Assessment of Number of Repeated Studies and Additional Hospitalization}

The manual-injection group had 14 of 45 patients (31\%) with repeated studies. In the automatic-injection group, 2 of 39 patients $(5 \%)$ required repeated studies. Using the $\chi^{2}$ test, we detected a statistically significant difference in the fraction of repeated studies between the groups $(P=$ 0.0025).

The additional length of hospital stay was longer in the manual-injection group than it was in the automaticinjection group and significantly different $(P=0.002)$ by Wilcoxon rank sum test. Fourteen of 45 patients in the manual-injection group had additional days of hospitalization (range, 0-7 d), compared with only 2 of 39 patients in the automatic-injection group (range, 0-1 d).

\section{Radiation Exposure}

On the basis of exposure data collected from radiation dosimetry reports, 7 of 15 nurses during the manualinjection period and 2 of 15 nurses during the automaticinjection period had greater than the minimal reporting doses (averages, $12.7 \mathrm{mrem}$ for manual injection and 13.1 mrem for automatic injection). These data yielded staff exposure rates (i.e., average dose per nurse $\times$ number of exposed nurses/number of patients or studies evaluated) of
8.1 mrem per study evaluation for manual injection and 1.1 mrem per study evaluation for automatic injection. Because there was a reduction of the number of repeated studies using automatic injection, the radiation exposure rate per patient was $11.1 \mathrm{mrem}$ with manual injection and $1.2 \mathrm{mrem}$ with automatic injection.

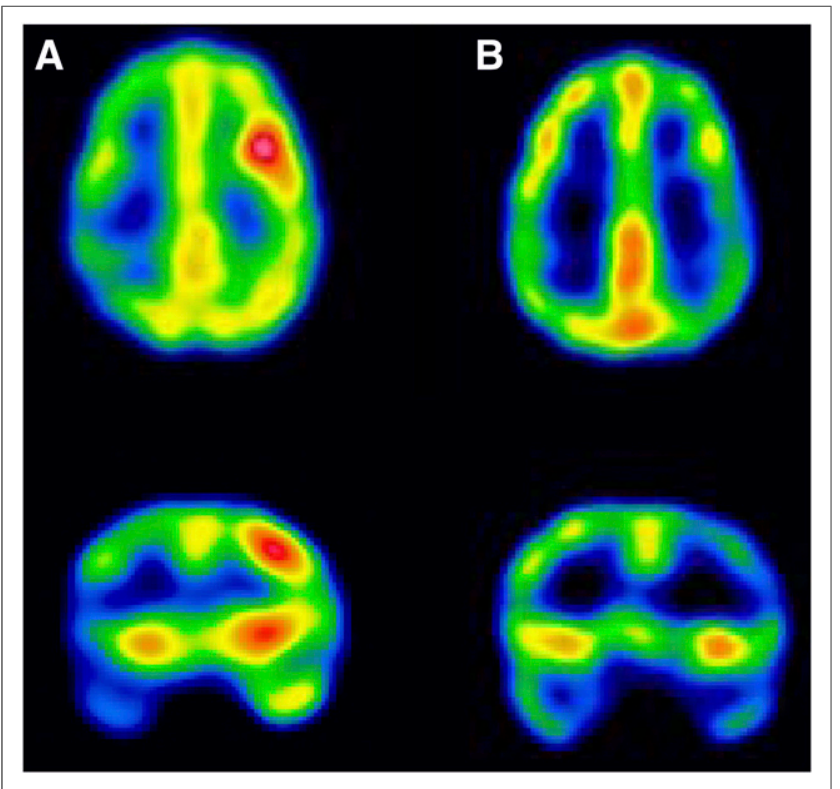

FIGURE 3. Ictal and interictal SPECT scans with automatic injection. Fifteen-year-old female with intractable seizure. (A) Ictal SPECT transaxial (upper) and coronal slice (lower) with latency time of $5 \mathrm{~s}$ and total duration of seizure of $8 \mathrm{~s}$ demonstrate relative focal hyperperfusion in left frontal lobe, compared with right side. (B) Interictal SPECT scans demonstrate focal hypoperfusion involving the posterior left frontal lobe. 


\section{DISCUSSION}

Using the first dedicated, commercially available, automatic injector approved by the Food and Drug Administration, this study demonstrated the clinical advantages of remotely controlled automated tracer injection, compared with manual injection, for ictal SPECT evaluation of pediatric epilepsy patients.

Although the previous studies mainly focused on the clinical feasibility of the custom-built automated injector for ictal SPECT, our study is the first, to our knowledge, to use a dedicated automatic injector system in a large population of children with epilepsy.

The use of an automatic injector, compared with a manual injector, significantly reduced latency time ( 8 $\mathrm{s}$ with automatic vs. $18 \mathrm{~s}$ with manual injection). A corresponding increase in localization rate (fraction of studies with a single localizing focus) from $49 \%$ (22/ $45)$ to $79 \%(31 / 39)(P=0.0075)$ was observed with the introduction of the automated injector. Our findings can be contrasted with the work of Setoain et al., in which no statistically significant difference in overall localization rate between the manual- and automatic-injection groups was identified $(65 \%$ and $78 \%$, respectively, $P=$ 0.14) (25).

Compared with a manual-injection system, in the automatic-injection group the presence of an additional intravenous line connecting the automatic injector pump and patients' injection site prolonged the time of delivery of tracer to the brain (additional time delay of $3.5 \mathrm{~s}$ with a tubing system of $7 \mathrm{~mL}$ of total volume). A benefit is that the additional intravenous line length allows more mobility to patients with hypermotoric symptoms. In our study, the average difference in latency time between the groups with and without the automatic injector was $10 \mathrm{~s}$, and this significant reduction in latency time can easily overcome the extra delay associated with an additional line in the automatic injector setup.

Although no statistical analysis was performed, the dosimetry results suggest that there was a reduction in radiation exposure rate after the automatic injector was introduced, presumably because an automatic-injection system lessens the need for nursing staff to closely handle the radiopharmaceuticals. In addition, there was a further reduction in radiation exposure rate per patient because of fewer repeated studies using the automatic injector.

The surgical outcome result could not be obtained for all patients in this study because in some cases surgery was not performed or there was insufficient time for follow-up after surgery if it was done. Therefore, the final concordance rate of localization of epileptogenic focus could not be discussed in this paper and remains a limitation of this study.

The automatic injector could be improved by incorporating the ability to administer a tracer volume that depends on the time-from-loading. With this feature, as the tracer decays, a proportionally greater volume could be injected, keeping the injected dose approximately constant, independent of injection time. Currently, the nursing staff manually reprograms the injector from time to time to accomplish this goal.

\section{CONCLUSION}

Our study demonstrated the benefit of using a dedicated automatic injector, compared with the manual-injection process, by decreasing latency time, resulting in an improvement in the degree of intensity of an epileptogenic focus. A decrease in latency time also results in a decrease in number of repeated studies and days of additional hospitalization and supports the clinical value of this automatic injector for the presurgical evaluation of ictal SPECT.

\section{DISCLOSURE}

The costs of publication of this article were defrayed in part by the payment of page charges. Therefore, and solely to indicate this fact, this article is hereby marked "advertisement" in accordance with 18 USC section 1734. No potential conflict of interest relevant to this article was reported.

\section{ACKNOWLEDGMENTS}

We thank Geraldine VonHofen for providing data on radiation exposure, Christopher Edwards for processing the images, and Crystal Doll for proofreading the manuscript.

\section{REFERENCES}

1. Engel J, Wiebe S, French J, et al. Practice parameter: temporal lobe and localized neocortical resection for epilepsy. Epilepsia. 2003;44:741-751.

2. McIntosh AM, Kalnins RM, Mitchell LA, Fabinyi GC, Briellman RS, Berkovic SF. Temporal lobectomy: long term seizure outcome, late recurrence, and risks for seizure recurrence. Brain. 2004;127:2018-2030.

3. Téllez-Zenteno JF, Dhar R, Wiebe S. Long-term seizure outcomes following epilepsy surgery: a systematic review and meta-analysis. Brain. 2005;128: 1188-1198.

4. Tonini C, Beghi E, Berg AT, et al. Predictors of epilepsy surgery outcome: a meta-analysis. Epilepsy Res. 2004;62:75-87.

5. Al-Kaylani M, Konrad P, Lazenby B, Blumenkopf B, Abou-Khalil B. Seizure freedom off antiepileptic drugs after temporal lobe epilepsy surgery. Seizure. 2007;16:95-98.

6. Asztely F, Ekstedt G, Rydenhag B, Malmgren K. Longterm follow up of the first 70 operated adults in the Gotenborg epilepsy surgery series with respect to seizures, psychosocial outcome and use of antiepileptic drugs. J Neurol Neurosurg Psychiatry. 2007;78:605-609.

7. Spencer SS, Berg AT, Vickrey BG, et al. Predicting long-term seizure outcome after resective epilepsy surgery. Neurology. 2005;65:912-918.

8. Zaatreh MM, Firlik KS, Spencer DD, Spencer SS. Temporal lobe tumoral epilepsy: characteristics and predictors of surgical outcome. Neurology. 2003;61: 636-641.

9. Holman BL, Devous MD Sr. Functional brain SPECT: the emergence of a powerful clinical method. J Nucl Med. 1992;33:1888-1904. 
10. Grünwald F, Durwen HF, Bockisch A, et al. Technetium-99m-HMPAO brain SPECT in medically intractable temporal lobe epilepsy: a postoperative evaluation. J Nucl Med. 1991;32:388-394.

11. Van Paesschen W. Ictal SPECT. Epilepsia. 2004;45(suppl4):35-40.

12. Newton MR, Berkovic SF, Austin MC, Rowe CC, McKay WJ, Alcorn PF. Ictal postictal and interictal single-photon emission tomography in the lateralization of temporal lobe epilepsy. Eur J Nucl Med. 1994;21:1067-1071.

13. Rowe CC, Berkovic SF, Sia ST, et al. Localization of epileptic foci with postictal single photon emission computed tomography. Ann Neurol. 1989; 26:660-668.

14. Spencer SS. The relative contributions of MR imaging, SPECT, and PET imaging in epilepsy. Epilepsia. 1994;35(suppl):72-89.

15. Devous MD Sr, Thisted RA, Morgan GF, Leroy RF, Rowe CC. SPECT brain imaging in epilepsy: a meta-analysis. J Nucl Med. 1998;39:285-293.

16. Kaminska A, Chiron C, Ville D, et al. Ictal SPECT in children with epilepsy: comparison with intracranial EEG and relation to postsurgical outcome. Brain. $2003 ; 126: 248-260$.

17. Koo CW, Devinsky O, Hari K, Balasny J, Noz ME, Kramer EL. Stratifying differences on ictal/interictal subtraction SPECT images. Epilepsia. 2003;44: 379-386.
18. Lee HW, Hong SB, Tae WS. Opposite ictal perfusion patterns of subtracted SPECT. Hyperperfusion and hypoperfusion. Brain. 2000;123:2150-2159.

19. Lee JD, Kim HJ, Lee BI, Kim OJ, Jeon TJ, Kim MJ. Evaluation of ictal brain SPET using statistical parametric mapping in temporal lobe epilepsy. Eur J Nucl Med. 2000;27:1658-1665.

20. Lee SK, Lee SY, Yun CH, Lee HY, Lee JS, Lee DS. Ictal SPECT in neocortical epilepsies: clinical usefulness and factors affecting the pattern of hyperperfusion. Neuroradiology. 2006;48:678-684.

21. Kazemi NJ, Worrell GA, Stead SM, et al. Ictal SPECT statistical parametric mapping in temporal lobe epilepsy surgery. Neurology. 2010;74: $70-76$.

22. Knowlton RC. The role of FDG-PET, ictal SPECT, and MEG in the epilepsy surgery evaluation. Epilepsy Behav. 2006;8:91-101.

23. Sepkuty JP, Lesser RP, Civelek CA, Cysyk B, Webber R, Shipley R. An automated injection system (with patient selection) for SPECT imaging in seizure localization. Epilepsia. 1998;39:1350-1356.

24. Feichtinger M, Eder H, Holl A, et al. Automatic and remote controlled ictal SPECT injection for seizure focus localization by use of a commercial contrast agent application pump. Epilepsia. 2007;48:1409-1413.

25. Setoain X, Pavía J, Serés E, et al. Validation of an automatic dose injection system for ictal SPECT in epilepsy. J Nucl Med. 2012;53:324-329. 